\title{
Parameterization of turbulent viscosity over orography
}

\author{
STEFAN EMEIS*
}

Institut für Meteorologie und Klimaforschung, Atmosphärische Umweltforschung (IMK-IFU),
Forschungszentrum Karlsruhe GmbH, Germany

(Manuscript received June 26, 2003; in revised form October 16, 2003; accepted October 24, 2003)

\begin{abstract}
Turbulent viscosity is an important parameter in numerical flow models that controls the vertical turbulent exchange. Vertical and temporal variations of turbulent viscosity have been determined from SODAR data taken over the Black Forest and the Alpine foreland in southern Germany in summer 2002. The turbulent viscosity - an analogon to the molecular viscosity - has been computed from the ratio between the variance of the vertical velocity and the mean shear of the horizontal wind. Turbulent viscosity shows a clear diurnal course with daytime peaks up to $1000 \mathrm{~m}^{2} / \mathrm{s}$. An orographical influence on this quantity becomes visible from the presented data. A first step towards a parameterization of turbulent viscosity with mountain-specific length and velocity scales is shown.
\end{abstract}

\begin{abstract}
Zusammenfassung
Die turbulente Viskosität ist ein wichtiger Parameter in numerischen Strömungssimulationsmodellen, die auch die Stärke des turbulenten Vertikalaustauschs des Impuls kontrolliert. Vertikale und zeitliche Variationen der turbulenten Viskosität sind hier aus SODAR-Daten bestimmt worden, die im Sommer $2002 \mathrm{im}$ Schwarzwald und im deutschen Alpenvorland gewonnen worden sind. Die turbulente Viskosität - eine Analogie zur molekularen Viskosität - ist dabei aus dem Quotienten von der Varianz der Vertikalgeschwindigkeit und der mittleren vertikalen Scherung des horizontalen Windes berechnet worden. Sie zeigt in den untersuchten Zeiträumen einen klaren Tagesgang mit Spitzenwerten tagsüber bis zu $1000 \mathrm{~m}^{2} / \mathrm{s}$. Der Einfluss der Orographie wird durch die generell etwas höheren Werte für die turbulente Viskosität über dem Schwarzwald sichtbar. Ein möglicher erster Parametrisierungsansatz für diese Viskosität als Funktion typischer orographiespezifischer Längen- und Geschwindigkeitsskalen wird vorgestellt.
\end{abstract}

\section{Introduction}

Mountainous regions are expected to contribute significantly to the export of polluted boundary-layer air into the free troposphere. The turbulent and convective processes involved differ from those found over flat terrain (see e.g. Founda et al., 1997 and many further references given therein). But parameterization of turbulent vertical exchange processes over mountainous terrain in meso-scale models is still based on turbulence data taken over flat terrain. In order to improve this situation, in particular the formulation of turbulent viscosity - an analogy to the molecular viscosity - is investigated here. The physical dimension of this viscosity is a product of a length and a velocity scale. The parameterization task is to find orography-related length and velocity scales which control the magnitude of turbulent viscosity in the boundary-layer over complex terrain.

The macro-scale interaction between flow and orography depends on the ratio between the length scale of the orography $\mathrm{L}$ and the internal length scale of the atmospheric flow (ADRIAN and FRÜHWALD 2002). In

\footnotetext{
*Author's address: Stefan Emeis, Institut für Meteorologie und Klimaforschung, Atmosphärische Umweltforschung (IMK-IFU), Forschungszentrum Karlsruhe $\mathrm{GmbH}$, Kreuzeckbahnstr. 19, D82467 Garmisch-Partenkirchen,e-mail: stefan.emeis@imk.fzk.de
}

a usually stably stratified atmosphere the latter is the length scale of internal gravity waves U / N (N: BruntVäisälä frequency, U scale of horizontal flow velocity). This ratio is known as Froude number $\mathrm{Fr}=\mathrm{U} / \mathrm{NL}$. Typical values of this internal length scale are in the order of $1000 \mathrm{~m}$. The macro-scale interaction is further modified by the ratio between the Rossby radius of deformation U / $\mathrm{f}$ (f: Coriolis parameter) and the length scale of the orography, expressed by a Rossby number Ro $=\mathrm{U} / \mathrm{fL}$. For Ro $<1$ and Fr $<1$ (i.e. $\mathrm{L}>100000 \mathrm{~m}$ ) we find leeward propagating waves, for Ro $>1$ and $\mathrm{Fr}<$ $1(100000 \mathrm{~m}>\mathrm{L}>1000 \mathrm{~m})$ we find vertically propagating lee waves, and for Ro $>1$ and $\mathrm{Fr}>1(\mathrm{~L}<1000 \mathrm{~m})$ we find once again leeward propagating gravity waves, which have a decreasing amplitude with decreasing $\mathrm{L}$.

The aforementioned frictionless influences of the orography on the air flow also lead to enhanced turbulence mainly via mechanical production by enhanced shear, see e.g. the turbulence produced in a foehn flow. Further, the turbulence over orography is enhanced due to thermally induced secondary circulations such as slope winds, valley and mountain winds, convection above crests and summits. For numerical flow models with grid constants larger than these secondary circulations also these thermally induced circulations are tur- 
Table 1: Possible length and velocity scales that could characterize the turbulent viscosity over complex terrain. Top: within a valley, bottom: in the boundary layer over orographically complex terrain.

\begin{tabular}{lll}
\hline layer & length scale & velocity scale \\
\hline valley boundary layer & $\begin{array}{l}\text { inversion height within the valley } \mathrm{L}_{i, V} \\
\text { width of the valley } \mathrm{L}_{V}\end{array}$ & speed of the valley wind $\mathrm{U}_{V}$ \\
& $\begin{array}{l}\text { mountain boundary layer } \\
\text { distances between two ridges } \mathrm{L}_{R}\end{array}$ & geostrophic wind speed $\mathrm{U}_{g}$ \\
\hline
\end{tabular}

bulent motions that have to be parameterized. A parameterized turbulence in numerical flow models acts like an additional viscosity $v_{t}$. Thus it seems to be natural to describe this turbulence in terms of a turbulent viscosity - an analogon to the molecular viscosity $v$. But there is one difference between molecular and turbulent viscosity that has to be kept in mind: whereas molecular viscosity is a material constant of the flowing medium, turbulent viscosity is a time and space dependent property of the flow itself.

The length scale of motions that makes a flow viscous is $v / U$. The ratio of the length scale $L$ of the orography with this viscous length scale is the Reynolds number $\mathrm{Re}=\mathrm{UL} / \mathrm{V}$. If we now introduce a turbulent viscosity $v_{t}$ (see equations (2.2) and (2.3) below for a precise definition) we can form a turbulent Reynolds number $\mathrm{Re}_{t}=\mathrm{UL} / \mathrm{v}_{t}$. This turbulent Reynolds number has proven to be a good means to scale the pressure drag exerted by mountains on a neutrally stratified flow in the atmospheric boundary layer. Theoretical scaling arguments gave (with a factor $\gamma$ that has to be determined experimentally and the height scale $\mathrm{H}$ of the orography) for the normalized pressure drag $c_{w}$ (EMEIS, 1990):

$$
c_{w}=\gamma\left(H^{2} / L^{2}\right) R e_{t}{ }^{-2 / 3}
$$

and numerical simulations with a meso-scale flow model (KAMM, ADRIAN and FIEDLER (1991)) of nearly neutrally stratified flows over two-dimensional sinusoidal ridges gave (EMEIS, 1990, 1994):

$$
c_{w}=39\left(H^{2.17} / L^{2.17}\right) R e_{t}{ }^{-2 / 3}
$$

Non-linear phenomena like lee eddies that appeared in the numerical simulations for steeper ridges are responsible for the increased dependence (2.17 instead of 2.0) of the pressure drag on the slope $(\mathrm{H} / \mathrm{L})$ of the ridges.

Returning to dimensional quantities and solving the numerically found equ. (1.2) for the turbulent viscosity yields (EMEIS, 1994):

$$
v_{t}=0.0007 U L
$$

Putting numbers into (1.3) gives a first indication of the expected magnitude of the turbulent viscosity. Assuming $20 \mathrm{~m} / \mathrm{s}$ for the mean horizontal velocity over the mountains $\mathrm{U}$ and $5000 \mathrm{~m}$ for the width of the mountain ridge $\mathrm{L}$ gives a turbulent viscosity of $70 \mathrm{~m}^{2} / \mathrm{s}$. This is much more than usually can be found at the top $(\mathrm{z} \approx$ $80 \mathrm{~m}$ ) of a Prandtl-layer with a friction velocity of about $u_{*}=0.5 \mathrm{~m} / \mathrm{s}$. There we just have $v_{t}=0.4 \mathrm{u}_{*} \mathrm{z}=16 \mathrm{~m}^{2} / \mathrm{s}$ (see also equ. (2.4) below) for neutral stratification, even less with stable stratification (about $3 \mathrm{~m}^{2} / \mathrm{s}$ for a MoninObukhov length $\mathrm{L}_{*}$ of $100 \mathrm{~m}$ ), and somewhat more under unstable conditions (about $30 \mathrm{~m}^{2} / \mathrm{s}$ for $\mathrm{L}^{*}=-100 \mathrm{~m}$ and about $45 \mathrm{~m}^{2} / \mathrm{s}$ for $\mathrm{L}_{*}=-20 \mathrm{~m}$ ). Tab. 1 gives some ideas how the orography dependent length and velocity scales could be chosen. With the choices from Tab. 1 the following combinations could be meaningful for Eq. (1.3):

$$
\begin{aligned}
& v_{t}=\alpha U_{V} L_{i, V} \\
& v_{t}=\alpha U_{V} L_{V} \\
& v_{t}=\alpha U_{g} L_{R}
\end{aligned}
$$

\section{Formulations for the turbulent viscosity}

The applicability of the above concept of turbulence viscosity and the possible parameterizations given in (1.41.6) can only be proven by an experiment that delivers data for the turbulent viscosity in flow over orography. The usual method to measure (molecular) viscosity (i.e. to calculate the viscosity using Stoke's law from the measured equilibrium fall speed of a sphere in the medium in question) cannot be performed in the real atmosphere. We therefore have to return to the defining equation, in which molecular viscosity $v$ is a factor of proportionality between the shear stress $\tau$ and the shear $\partial u / \partial z$ in a laminar flow (with the density of the medium $\rho$ and the flow speed $u$ in mean flow direction):

$$
\tau=v \rho \partial u / \partial z
$$

In (2.1) molecular viscosity is a function of the temperature of the medium only. As shear stress and shear always have the same sign this viscosity is a positive quantity. Transferring this relation to a turbulent flow yields an analogous equation for the turbulent viscosity $v_{t, i j k l}($ with $\mathrm{i}, \mathrm{j}, \mathrm{k}, \mathrm{l}=1 . .3$ ):

$$
-\rho \overline{u_{i}^{\prime} u_{j}^{\prime}}=v_{t, i j k l} \rho \partial u_{k} / \partial x_{l}
$$


with $\mathrm{u}_{1}^{\prime}$ being the longitudinal (along the mean flow direction), $\mathrm{u}_{2}^{\prime}$ the lateral, and $\mathrm{u}_{3}^{\prime}$ the vertical flow speed fluctuations. For the sake of simplicity and readability we will write $\mathrm{u}$ instead of $\mathrm{u}_{1}, \mathrm{v}$ instead of $\mathrm{u}_{2}$, and $\mathrm{w}$ instead of $u_{3}$ in the following. Likewise we will use $x$ instead of $x_{1}$ and $z$ instead of $x_{3}$. Eq. (2.2) demonstrates that - in principle - turbulent viscosity is a fourth-order tensor with 81 components. In the following only the component of this tensor that corresponds to the coefficient of vertical turbulent exchange of momentum in numerical models (in the field of numerical modelling this component is usually called 'turbulent exchange coefficient' $\mathbf{K}_{m, v}$ ) will be addressed as turbulent viscosity $v_{t}$.

$$
K_{m, v}=v_{t}=-\frac{\overline{u^{\prime} w^{\prime}}}{\frac{\partial u}{\partial z}}
$$

As the vertical turbulent momentum flow is much larger than the mean vertical momentum flow, this is the most important component that has to be parameterized in the simulation of turbulent boundary layer flow. Applying (2.3) to a homogeneous Prandtl-layer (i.e. putting $\mathrm{u}_{*}^{2}$ for $\overline{u^{\prime} w^{\prime}}$ and $\mathrm{u}_{*} / \kappa z$ for $\frac{\overline{\partial u}}{\partial z}$, with the von Kármán constant $\kappa=0.4$ ) leads to the well-known expression for the turbulent exchange coefficient $\mathrm{K}_{m, v}\left(=\mathrm{v}_{t}\right)$ that increases linearly with height if the wind speed increases logarithmically with height:

$$
v_{t}=u_{*} \kappa z
$$

Using (2.3), we can determine the turbulent viscosity in a real flow if we measure the turbulent momentum flux $\overline{u^{\prime} w^{\prime}}$ and the shear $\frac{\overline{\partial u}}{\partial z}$ as it has been done by CAMPISTRON et al. (1991). While the shear as a mean quantity is relatively easily available from measurements, it is very difficult to get the turbulent momentum flux from remote sensing data (this is the type of data available for this study). Looking for a surrogate for the turbulent momentum flux we find from STULL (1988) or e.g. from data presented in EMEIS et al. (1995) that $\sigma_{w}^{2}$ and $\overline{u^{\prime} w^{\prime}}$ are proportional in the surface layer. In the case of near neutral and stable thermal stratification of the air over homogeneous terrain we find:

$$
\sigma_{w}^{2}=1.6 \overline{u^{\prime} w^{\prime}}
$$

Inserting this proportionality into (2.3) we get:

$$
v_{t}=\frac{\sigma_{w}^{2}}{1.6 \frac{\partial u}{\partial z}}
$$

(2.6) is valid only for a near neutral or stable surface layer. Above this layer the situation is more complicated. For a near neutral mixed layer where mechanical production of turbulence dominates, both, $\sigma_{w}$ and

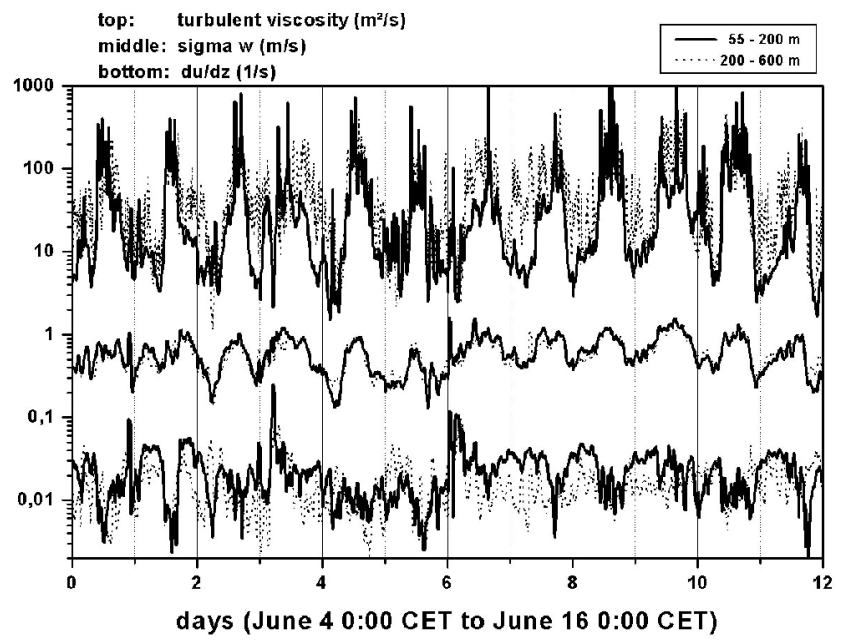

Figure 1: 12-day time series of turbulent viscosity (top, in $\mathrm{m}^{2} / \mathrm{s}$ ), $\mathrm{sw}$ (middle, in $\mathrm{m} / \mathrm{s}$ ), and vertical shear (bottom, in $1 / \mathrm{s}$ ) from sodar data at Zwickgabel (Black Forest, Southern Germany) in June 2002. Data are shown for two height intervals: full line: 55-200 m, dashed line: 200-600 m above ground. On June 10 sunrise was at 4:22 CET, sunset at 20:29 CET.

the turbulent momentum flux, decrease with height. Using again information from STULL (1988, his equations 9.5.2b, 9.5.3f, and 9.5.3j) we get with the mixed layer height $\mathrm{z}_{i}$ :

$$
\sigma_{w}^{2}=1.6\left(1-z / z_{i}\right)^{-0.5} \overline{u^{\prime} w^{\prime}}
$$

which if the factor 1.6 from $9.5 .3 \mathrm{j}$ is used in $9.5 .3 \mathrm{f}$ converges to (2.5) for $\mathrm{z}$ against zero. For $\mathrm{z}=0.5 \mathrm{z}_{i}$ the factor between $\sigma_{w}^{2}$ and $\overline{u^{\prime} w^{\prime}}$ is 2.26 , for $\mathrm{z}=0.9 \mathrm{z}_{i}$ it is 5.06. By inserting (2.7) into (2.3) this leads to an approximation for $\mathrm{v}_{t}$ :

$$
v_{t}=\frac{\sigma_{w}^{2}}{1.6\left(1-z / z_{i}\right)^{-0.5} \overline{\frac{\partial u}{\partial z}}}
$$

(2.8) should not be used very close to the top of the mixed layer. For a stable mixed layer, equations 9.4.2c and 9.4.3f in STULL (1988) suggest a more or less height independent, constant ratio between $\sigma_{w}^{2}$ and $\overline{u^{\prime} w^{\prime}}$ of the order 2.5. Thus, a rough compromise between this finding, eq. (2.6), and eq. (2.8) is to use

$$
v_{t}=\frac{\sigma_{w}^{2}}{a \frac{\partial u}{\partial z}}
$$

in the following with a height dependent a. We will put a $=1.6$ in a lower layer that includes the surface layer (we will choose 55-200 $\mathrm{m}$ for this layer in the next chapter), $\mathrm{a}=2.0$ for a middle layer $(200-600 \mathrm{~m})$, and $\mathrm{a}=2.5$ for an upper layer (600-1000 m). The approximation (2.9) to turbulent viscosity (2.3) has been made because (2.9) can be computed from sodar data without the need of 


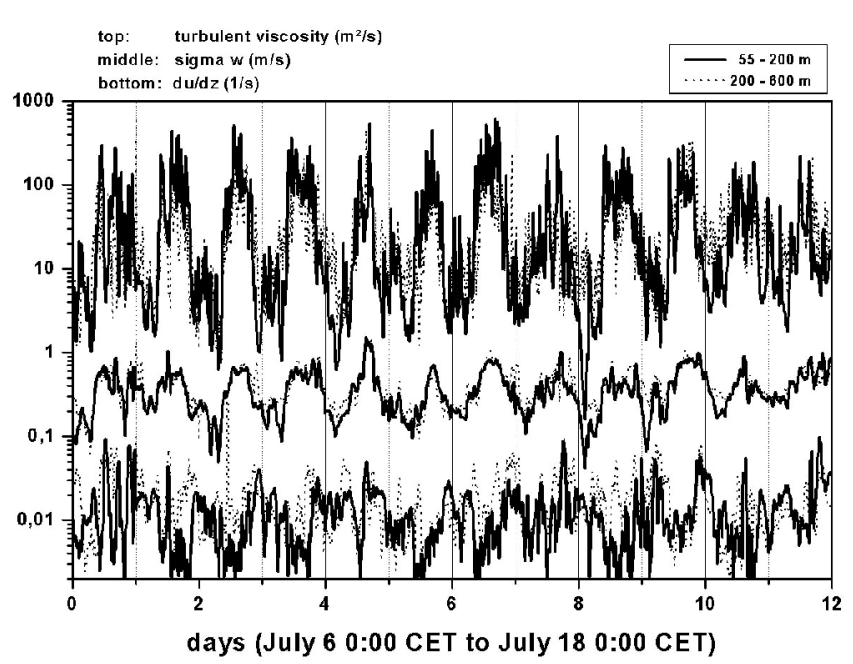

Figure 2: As Fig. 1, but for Königsdorf (Alpine foreland, Southern Germany) in July 2002. On July 12 sunrise was at 4:26 CET, sunset at 20:12 CET.

additional information. $\overline{u^{\prime} w^{\prime}}$ cannot be computed from sodar measurements. This offers the opportunity to determine the diurnal course and the vertical structure of turbulent viscosity and to estimate the range of values this quantity can take from longer time series of groundbased measurements with a sodar. Because we have used a quadratic expression $\left(\sigma_{w}^{2}\right)$ instead of $\overline{u^{\prime} w^{\prime}}$ in (2.9), the information about the sign of the turbulent momentum flux is lost in (2.9). In order to assure the positiveness of the turbulent viscosity only the absolute value of the shear must be used in (2.9).

\section{Experiment}

In an experiment that took place in the Black Forest and in the Alps in June and July 2002 a METEK DSDR 3x 7 sodar (REITEBUCH and EMEIS, 1998) was operated to monitor temporal and spatial variations of wind and turbulence during days with convection and thunderstorms over the Black Forest in June 2002 and the Alpine foreland in July 2002. The experiment was part of the activities within the project VERTIKATOR of the German research programme AFO2000. The sodar measures u, $\mathrm{v}, \mathrm{w}$, and $\sigma_{w}$ quite well. $\sigma_{u}$ and $\sigma_{v}$ are also available but are not reliable.

Sodar wind data (all three components of the wind and $\sigma_{w}$ ) were recorded with $10 \mathrm{~min}$ temporal and 30 $\mathrm{m}$ vertical resolution. Lowest measurement height was centred at $55 \mathrm{~m}$, the range of the instrument is about $1000 \mathrm{~m}$. In order to smooth the results turbulent viscosity was calculated from the sodar data using a moving average over 6 time steps $(60 \mathrm{~min})$ and three height intervals $(90 \mathrm{~m})$. Assuming a wind speed of $10 \mathrm{~m} / \mathrm{s}^{-1}$ (most times the wind speeds were lower) and applying Taylor's hypothesis leads to the interpretation that sodar wind data averaged over $10 \mathrm{~min}$ represent a horizontal mean over about $6 \mathrm{~km}$. If thermally induced convection is fixed to close-by slopes orientated towards the sun, the $10 \mathrm{~min}$ average wind data has to be interpreted more locally.

In the Black Forest (a mountain range in southwestern Germany with medium slopes and peak heights between 1000 and $1500 \mathrm{~m}$ asl) the sodar was operated in a height of $860 \mathrm{~m}$ asl at Zwickgabel near Schönmünzach at a saddle point on a crest line. Mountain slopes are quite steep around the measurement site. Adjacent valleys are about 5 to $10 \mathrm{~km}$ wide and about $500 \mathrm{~m}$ deep. The mountains in this area are completely covered with forest. In the Alpine foreland it was operated in a height of $600 \mathrm{~m}$ asl at the glider airport Königsdorf near Bad Tölz south of Munich. The airport is situated in open, nearly flat terrain with patches of forest about $20 \mathrm{~km}$ north of the Alpine foothills, which rise to peak heights between 1500 and $3000 \mathrm{~m}$. We expect that the flow at the Black Forest site is considerably influenced by the nearby mountains and valleys while the flow at the Alpine foreland site will be representative for a more homogeneous terrain.

\section{Results}

From (2.9) we expect to find high turbulent viscosity in cases with a high variance of the vertical velocity and low vertical shear. This should mainly happen during days with thermally induced convection. On the other hand, turbulent viscosity should be smallest during stable nights with nearly vanishing $\sigma_{w}^{2}$ and large vertical velocity gradients.

Fig. 1 shows vertically averaged time series of turbulent viscosity computed from (2.9), $\sigma_{w}$, and $\frac{\overline{\partial u}}{\partial z}$ over the Black Forest for a period of 12 days from June 4, 0:00 CET to June 16, 0:00 CET (at this site CET is about 26 min ahead of the mean local time) for the height ranges from 55 to $200 \mathrm{~m}$ and 200 to $600 \mathrm{~m}$. Fig. 2 shows the same quantities over the Alpine foreland for a period of 12 days from July 6, 0:00 CET to July 18 (here CET is about 14 min ahead of the mean local time). The range of values for the turbulent viscosity extends from 0 to about $1000 \mathrm{~m}^{2} / \mathrm{s}$ over the Black Forest and from 0 to about $600 \mathrm{~m}^{2} / \mathrm{s}$ over the Alpine foreland.

The most prominent feature in both figures is the daily cycle of the magnitude of the turbulent viscosity with a maximum around noon and a minimum around midnight. This daily cycle is not only due to such a cycle in $\sigma_{w}$ but especially in clear, calm nights it is also due to increased wind shear. This indicates that the overall thermal stability of the air is primarily controlling the magnitude of turbulent viscosity.

Tab. 2 gives the mean values and standard deviations of the three quantities plotted in Figures 1 and 2. 
Table 2: Mean and standard deviation of turbulent viscosity (in $\mathrm{m}^{2} / \mathrm{s}$ ), variance of vertical velocity (in $\mathrm{m} / \mathrm{s}$ ), and vertical shear (1/s) for three different height intervals and four time periods (two from Black Forest and two from Alpine foreland). June 10-13 was a very windy period and July 7-9 was dominated by convection.

\begin{tabular}{lrrrrrrr}
\hline & & \multicolumn{2}{c}{$55-200 m$} & \multicolumn{2}{c}{$200-600 m$} & \multicolumn{2}{c}{$600-1000 m$} \\
& & mean & st. dev. & mean & st. dev. & mean & st. dev. \\
\hline \multirow{3}{*}{ Black Forest } & $v_{t}$ & 48.61 & 102.40 & 57.51 & 67.90 & 28.40 & 39.56 \\
June 4-16 & $\sigma_{w}$ & 0.60 & 0.30 & 0.59 & 0.26 & 0.55 & 0.23 \\
& $\frac{\partial u}{\partial z}$ & 0.022 & 0.019 & 0.018 & 0.013 & 0.038 & 0.047 \\
& & & & & & & \\
Black Forest & $v_{t}$ & 54.86 & 130.29 & 75.10 & 80.08 & 41.31 & 48.31 \\
June 10-13 & $\sigma_{w}$ & 0.79 & 0.28 & 0.73 & 0.25 & 0.67 & 0.21 \\
& $\frac{\partial u}{\partial z}$ & 0.029 & 0.016 & 0.016 & 0.012 & 0.035 & 0.070 \\
Alpine & & & & & & & \\
foreland & $v_{t}$ & 44.95 & 70.56 & 32.26 & 46.57 & 13.60 & 21.61 \\
July 6-18 & $\frac{\sigma_{w}}{\partial u}$ & 0.38 & 0.22 & 0.42 & 0.22 & 0.42 & 0.16 \\
& & 0.014 & 0.013 & 0.020 & 0.020 & 0.040 & 0.027 \\
Alpine & $v_{t}$ & 56.56 & 80.87 & 33.78 & 33.78 & 7.58 & 9.52 \\
foreland & $\sigma_{w}$ & 0.37 & 0.19 & 0.41 & 0.20 & 0.38 & 0.14 \\
July 7-9 & $\frac{\partial u}{\partial z}$ & 0.011 & 0.009 & 0.021 & 0.029 & 0.049 & 0.038 \\
\hline
\end{tabular}

Here also the height range between 600 and $1000 \mathrm{~m}$ is included that has not been plotted in Figures 1 and 2 for the sake of clarity. From the values for the turbulent viscosity in Tab. 2 it is quite obvious that the layer with high turbulent viscosity reaches higher up over the Black Forest crest (up to $1000 \mathrm{~m}$ above the crest or $1860 \mathrm{~m}$ asl) than over the Alpine foreland (up to about $600 \mathrm{~m}$ above ground or $1200 \mathrm{~m}$ asl). There are probably several reasons for this fact. The three most prominent are: (1) the overall wind speed was somewhat higher over the Black Forest crest than over the Alpine foreland (it is very likely that this is mainly caused by the more exposed measuring site in the Black Forest and by the speed up over the mountain crest), (2) convective updrafts during daytime were stronger over the Black Forest slopes than over the flat Alpine foreland, and (3) due to the general flow pattern of Alpine pumping (LUGAUER and WinkLer 2003). Due to Alpine pumping (that occurs with clear and sunny weather only) we have in the Alpine foreland during daytime a shallower boundary layer with winds towards the Alps and a layer with mean sinking motion and weak winds away from the Alps above this boundary layer. The explanations (2) and (3) are especially supported by the fact that turbulent viscosity was extremely low in the upper height range (only one eighth to one fifth of the values in the lower two layers) during the convective period in the Alpine foreland during July 7-9 (see fourth and last entry in Tab. 2).
Another difference between Black Forest and Alpine foreland is that the maximum of the turbulent viscosity is in the lowest layer over the Alpine foreland and in the middle layer over Black Forest. This fact is most pronounced during the windy period June 10-13. The reason for this can be found in the large value of the shear in the lowest layer. For the Black Forest measuring site Tab. 2 gives always a higher value for the shear in the lowest layer than in the middle layer. This is probably due to the speed-up of the wind over the crest of the Black Forest during windy days that causes increased shear close to the ground and reduced shear aloft. Over the Alpine foreland the shear in the lowest layer is smaller than in the layer above. This feature is most prominent during the convective period July 7-9. During these days winds are generally weak close to the ground which results in very small shear in the lowest layer.

\section{Conclusions and outlook}

For the first time a rough estimate of temporal and vertical variation of turbulent viscosity over two types of complex terrain has been derived from sodar data. CAMPISTRON et al. (1991) used a radar to derive the turbulent viscosity in and over a valley. First of all especially at the Black Forest site the daytime turbulent viscosity was much larger than the value of $45 \mathrm{~m}^{2} / \mathrm{s}$ that one could expect at the top of a horizontally homogeneous but thermally unstable Prandtl layer $\left(\mathrm{z}=80 \mathrm{~m}, \mathrm{u}_{*}\right.$ 
$\left.=0.5, \mathrm{~L}_{*}=-20 \mathrm{~m}\right)$. This is mainly due to thermal production of turbulence over the slopes and due to larger vertical wind shear over the crests of the Black Forest.

The orographic influence on this quantity has become visible in two aspects:

- Peak values of turbulent viscosity tend to be larger over the horizontally very inhomogeneous Black Forest than over the nearly flat Alpine foreland because of the strong convection over the slopes of the Black Forest. This is seen less pronounced from the mean values given in Tab. 2 because the daytime maxima of $v_{t}$ are broader over the Alpine foreland than over the Black Forest. High $v_{t}$-values prevail over the Alpine foreland during the whole period of the daytime wind regime with nearsurface winds towards the mountains. This wind regime characterized by high thermally induced turbulence lasts longer than the regime of daytime convection over the slopes of the Black Forest.

- The turbulent boundary layer with high turbulent viscosity is considerably deeper over the crests of the Black Forest than over the Alpine foreland.

The turbulent viscosity data derived here from sodar measurements agree quite well with the findings of CAMPISTRON et al. (1991) from radar data. As in CAMPISTRON et al. we find the maximum values in the vertical profiles of turbulent viscosity over the Black Forest 400 to $500 \mathrm{~m}$ above ground. Our mean values for $v_{t}$ (Tab. 2) are somewhat lower than those given by CAMPISTRON et al. because we have computed a 12 day mean and CAMPISTRON et al. just a 6 hour-mean during a storm episode. Our peak values are higher than CAMPISTRON et al.'s values due to thermal instabilities during daytime, while their values were taken in winter during overcast conditions. In CAMPISTRON et al. the layer with enhanced turbulent viscosity is about one kilometre deep. Our measurements at the Black Forest site show enhanced turbulent viscosity at least over the whole range of the sodar data $(1000 \mathrm{~m})$, but a more shallow layer of about $600 \mathrm{~m}$ thickness over the Alpine foreland. On the other hand we cannot check the anisotropy that had been found by CAMPISTRON et al. in the valley for two reasons. First of all at the Black Forest site we have measured on top of a crest line and not within a valley, and secondly due to the approximation (2.9) we cannot distinguish between the longitudinal and the lateral component of the viscosity.

Turbulent viscosity (as given by Eq. (2.3)) is an important parameter in numerical flow models. It controls the strength of turbulent vertical exchange of heat, momentum, water vapour and other trace substances. Therefore its magnitude is decisive when computing the handover from near-surface layers to the upper parts of the atmospheric boundary layer and the free troposphere. The present study has shown that the first estimation for this quantity over mountainous terrain (Eq. (1.3)) is at least in the right order of magnitude.
The task still to do is to find the right length and velocity scales that have to enter into Eq. (1.3). For this purpose also the aircraft measurements from the VERTIKATOR experiment that took place over different types of mountainous terrain have to be evaluated in the near future. Eq. (1.3) has to be expanded, too. The present study has shown that turbulent viscosity has a strong daily cycle. Therefore the now constant factor 0.0007 must depend on the thermal state of the atmosphere, maybe on the Rayleigh number or something equivalent. The final proof for all these parameterizations will be to enter such a scheme into a numerical flow model and to show that better flow simulations and budgets of atmospheric trace substances over mountainous terrain are possible.

\section{Acknowledgements}

VERTIKATOR was funded by the German Ministry for Education and Research (BMBF) within the framework of the research programme AFO2000. I thank Peter WINKLER for helpful discussions on the subject of this paper and Philippe DROBINSKI and Rich COULTER for very helpful comments and questions during the review process.

\section{References}

AdriAn, G., F. FiEDLER, 1991: Simulation of unstationary wind and temperature fields over complex terrain and comparison with observations. - Beitr. Phys. Atmosph. 64, $27-$ 48.

Adrian, G., D. FrüHWAld, 2002: Design der neuen Modellkette GME/LM. - Promet 27, 106-110. (In German)

Campistron, B., A.W. Huggins, , A.B. Long, 1991: Investigations of a winter mountain storm in Utah. Part III: Single-Doppler radar measurements of turbulence. - J. Atmos. Sci. 48, 1306-1318.

EMEIS, S., 1990: Pressure drag of obstacles in the atmospheric boundary layer. - J. Appl. Meteorol. 29, 461-476.

—, 1994: Bestimmung und Parametrisierung des Druckwiderstands an Hindernissen in der atmosphärischen Grenzschicht. - Ber. Deut. Wetterd. 191, 189 pp. (In German)

EMEIS, S., H.P. FRANK, F. FIEDLER, 1995: Modification of air flow over an escarpment - results from the Hjardemål Experiment. - Bound.-Lay. Meteorol. 74, 131-161.

Founda, D., M. Tombrou, D.P. LALAS, D.N. AsIMAKOPOULOS, 1997: Some measurements of turbulence characteristics over complex terrain. - Bound.-Lay. Meteorol. 83, 221-245.

Lugauer, M., P. Winkler, 2003: submitted to Meteorol. Z.

REITEBUCH, O., S. EMEIS, 1998: SODAR-measurements for atmospheric research and environmental monitoring.Meteorol.Z., N. F. 7, 11-14.

STULL, R.B., 1988: An Introduction to Boundary Layer Meteorology. - Kluwer, 666 pp. 